\title{
Tratamiento de aguas residuales contaminadas con glifosato basado en el desarrollo de SBA- 15 impregnados con Fe como catalizadores avanzados para el proceso de oxidación húmeda en condiciones ambientales.
}

Treatment of glyphosate contaminated wastewater based on the development of Fe doped SBA-15 as advanced catalysts for wet oxidation process under room conditions.

\section{Eliana G. Vaschetto}

Centro de Investigación y Tecnología Química (CITeQ), Universidad Tecnológica Nacional (UTN), Consejo Nacional de Investigaciones Científicas y Técnicas (CONICET) - Argentina.

elivaschetto@hotmail.com

\section{María C. Gómez}

Centro de Investigación y Tecnología Química (CITeQ), Universidad Tecnológica Nacional (UTN), Consejo Nacional de Investigaciones Científicas y Técnicas (CONICET) - Argentina. m.cande.gomez@gmail.com

\section{Pablo A. Ochoa Rodríguez}

Centro de Investigación y Tecnología Química (CITeQ), Universidad Tecnológica Nacional (UTN), Consejo Nacional de Investigaciones Científicas y Técnicas (CONICET) - Argentina. pochoa@frc.utn.edu.ar

\section{Sandra G. Casuscelli}

Centro de Investigación y Tecnología Química (CITeQ), Universidad Tecnológica Nacional (UTN), Consejo Nacional de Investigaciones Científicas y Técnicas (CONICET) - Argentina. scasuscelli@frc.utn.edu.ar

\section{Verónica R. Elías}

Centro de Investigación y Tecnología Química (CITeQ), Universidad Tecnológica Nacional (UTN), Consejo Nacional de Investigaciones Científicas y Técnicas (CONICET) - Argentina. veroelias@gmail.com

\section{Griselda A. Eimer}

Centro de Investigación y Tecnología Química (CITeQ), Universidad Tecnológica Nacional (UTN), Consejo Nacional de Investigaciones Científicas y Técnicas (CONICET) - Argentina.

geimer@frc.utn.edu.ar 


\title{
Resumen
}

Se desarrollaron sólidos nanoestructurados impregnados con diferentes contenidos de hierro $(1 ; 2,5 ; 5$ y $10 \%$ p/p) como catalizadores eficaces para degradar soluciones acuosas de glifosato en condiciones de reacción extremadamente suaves: presión atmosférica y temperatura ambiente. Estos materiales se caracterizaron por XRD, fisisorción de $\mathrm{N}_{2}$, UVvis-DR y XPS. Se obtuvieron estructuras mesoporosas regulares típicas de los sólidos SBA-15 y se pudo ajustar la especiación de Fe variando la carga nominal del metal. Los catalizadores fueron evaluados en la reacción de degradación-fragmentación de glifosato mediante oxidación húmeda catalítica con aire, logrando niveles de degradación del herbicida del orden del 80\%. Se propuso un camino de reacción basado en la formación de un intermediario oxo-hierro (V) altamente reactivo a partir del complejo Fe-glifosato. De esta manera, se presenta una interesante tecnología con menor impacto ambiental y mayor sustentabilidad para la remediación de aguas contaminadas con glifosato.

Palabras clave: Tratamiento de aguas contaminadas, Glifosato, Nanomateriales, Contenido de Fe.

\begin{abstract}
Nanostructured solids doped with various iron contents (1, 2.5, 5 and 10wt.\%) were developed as efficient catalysts to degrade glyphosate aqueous solutions under extremely mild reaction conditions, atmospheric pressure and room temperature. These materials were characterized by XRD, $\mathrm{N}_{2}$ physisorption, UVvis-DR and XPS. Regular mesoporous structures typical of SBA-15 solids were obtained and the Fe speciation could be tuned by adjusting the metal nominal loading. The catalysts were evaluated in the glyphosate degradation-fragmentation reaction by catalytic wet oxidation with air, achieving herbicide degradation levels of the order of $80 \%$. A reaction way based on the formation of a highly reactive oxo-iron (V) intermediary from Fe-glyphosate complex was proposed. In this manner, an interesting technology with lower environmental impact and greater sustainability for the remediation of water polluted with glyphosate is presented.
\end{abstract}

Keywords: Polluted water treatment, Glyphosate, Nanomaterials, Fe content.

\section{Introducción}

Los herbicidas son sustancias químicas que se utilizan para eliminar las hierbas no deseadas de los cultivos. Los que se aplican directamente al suelo se denominan pre-emergentes y matan a la planta a medida que germina. Mientras tanto, los post-emergentes destruyen la planta cuando se aplican a sus hojas u otras partes una vez que ya ha germinado. El herbicida post-emergente más utilizado en todo el mundo es el glifosato $\left(\mathrm{C}_{3} \mathrm{H}_{8} \mathrm{NO}_{5} \mathrm{P}\right)$, que es el nombre común de la $\mathrm{N}$-fosfonometilglicina. Es de amplio espectro, no selectivo, con fitotoxicidad en plantas anuales y perennes. Debido a los diferentes métodos de aplicación de este herbicida y a las diversas condiciones ambientales (lluvias, vientos, etc.), una cantidad significativa del mismo aplicado a la planta logra llegar al suelo y desde allí se propaga a aguas subterráneas, en algunos casos hasta aguas superficiales o generando de una u otra manera contaminación. El destino del glifosato a través del suelo depende en gran medida de su solubilidad en agua (Dubois et al., 2011), de su capacidad para formar complejos con metales y de cómo se adsorbe en los componentes del suelo (Barja et al., 2001).

En este contexto, los procesos avanzados de oxidación se proponen como una alternativa de degradación muy prometedora para este tipo de compuestos en medios acuosos (Robert 
et al., 2002). Los procesos de oxidación húmeda con aire u $\mathrm{O} 2$ tienen el potencial de degradar contaminantes orgánicos tóxicos y/o refractarios, pero a altas temperaturas $\left(\sim 180-350^{\circ} \mathrm{C}\right)$ y presiones ( 20-200 atm) según Kyoung-Hun et al., 2011. Se ha demostrado que la adición de un catalizador sólido al sistema puede promover la formación de radicales en la superficie, acelerar la velocidad de reacción y mejorar la eficiencia, reduciendo drásticamente la severidad de las condiciones operativas. Así, los catalizadores modificados con metal pueden promover la formación de complejos, mejorando la eficiencia de los procesos de adsorción y degradación de fosfonatos, como el glifosato. Los silicatos mesoporosos como el SBA-15 aparecen como soportes catalíticos muy prometedores (Elías et al., 2020a) debido a su estructura porosa uniforme, alta área específica y la posibilidad de modificar químicamente su superficie con funciones específicas.

En este trabajo, se desarrollaron sólidos mesoestructurados modificados con diversos contenidos de hierro $(1 ; 2,5 ; 5$ y $10 \%$ p/p) mediante impregnación directa del material silíceo puro y además se evaluaron en la degradación de soluciones acuosas de glifosato por oxidación catalítica húmeda con aire en condiciones ambientales.

\section{Experimental}

\section{Síntesis de materiales}

Los catalizadores se sintetizaron usando Pluronic P123 como agente plantilla de la estructura disuelto en una solución $2 \mathrm{M}$ de $\mathrm{HCl}$. Posteriormente, se añadió la fuente de Si (Tetraetilortosilicato) y se ajustó el $\mathrm{pH}$ a 3,5 con una solución de $\mathrm{NH}_{4} \mathrm{OH}$. El gel obtenido se envejeció a $40^{\circ} \mathrm{C}$ durante $20 \mathrm{~h}$ y luego a $80^{\circ} \mathrm{C}$ durante $48 \mathrm{~h}$, sin agitación. El sólido resultante se filtró, lavó y secó a $60^{\circ} \mathrm{C}$ durante la noche. Luego, el material se calcinó a $500{ }^{\circ} \mathrm{C}$ durante 6 h con una rampa de $1^{\circ} \mathrm{C} /$ min y se identificó como SBA-15. La impregnación con Fe se llevó a cabo utilizando $\mathrm{FeCl}_{3} \cdot 6 \mathrm{H}_{2} \mathrm{O}$ disuelto en etanol con los siguientes contenidos nominales de Fe: $1 ; 2,5 ; 5$ y $10 \%$ p/p. Finalmente, los materiales se calcinaron a $350{ }^{\circ} \mathrm{C}$ durante $3 \mathrm{~h}$. Los sólidos se identificaron como $\mathrm{Fe} / \mathrm{SBA}(\mathrm{x})$, donde $\mathrm{x}$ representa el porcentaje en peso nominal de $\mathrm{Fe}$ impregnado.

\section{Caracterización de materiales}

Los materiales se caracterizaron estructuralmente por DRX (difractómetro Philips PW 3830 en el rango de $2 \theta$ desde $1,3^{\circ}$ a $80^{\circ}$ ). Se realizó un ajuste de perfil a cada máximo en el rango de ángulo alto y el tamaño medio del cristal (D) de la fase correspondiente se estimó utilizando la fórmula de Scherrer (1):

$$
D=\frac{0.9 \lambda}{\beta \cos \theta}
$$

donde $\beta$ (en radianes) es la amplitud intrínseca máxima después de restar la contribución instrumental, $\lambda$ es la longitud de onda de los rayos $\mathrm{X}$ y $\theta$ es el ángulo de Bragg (Elías et al., 2012), (Patterson, 1939). Cabe aclarar que se utilizó la ecuación de Scherrer para realizar una estimación aproximada del tamaño de los cristales.

Para determinar el área específica, se realizaron mediciones de fisisorción de nitrógeno en un pulso de punto único Chemisorb a $\mathrm{P} / \mathrm{P}_{0}=0,3$ y se aplicó el método $\mathrm{BET}$.

El contenido final de hierro se determinó aplicando el método colorimétrico de o-fenantrolina (3500-Fe) por espectrofotometría a $510 \mathrm{~nm}$ utilizando un espectrómetro Jasco 650 (Greenberg et al., 1992). El método se basa en la sensibilidad de la o-fenantrolina para formar 
un complejo con los iones $\mathrm{Fe}^{+2}$ presentes en solución. Por lo tanto, la absorción a $510 \mathrm{~nm}$ es proporcional a la concentración de iones $\mathrm{Fe}^{+2}$ solubilizados. Previo a este análisis, los sólidos se disgregaron con ácidos y la solución de cada muestra se trató con un agente reductor para convertir todos los iones $\mathrm{Fe}^{+3}$ presentes en $\mathrm{Fe}^{+2}$; finalmente, se midió la concentración de $\mathrm{Fe}^{+2}$, que puede considerarse como la concentración total de hierro presente en los sólidos disgregados (Greenberg et al., 1992), (Neyens et al., 2003).

Para la especiación de hierro en función del contenido de metal, se registraron espectros de reflectancia difusa UVvis (UVvis DR) usando un espectrómetro Jasco 650 con una esfera integradora en el rango de longitud de onda de 200-900 nm. Los espectros originales obtenidos fueron ajustados por varias bandas gaussianas utilizando el método convencional de mínimos cuadrados. Los cálculos de ajuste de curvas fueron útiles para determinar la ubicación de cada banda y el área relativa, con niveles de confianza dados por $\mathrm{R}^{2} \geq 0,99$ y $\chi^{2}<0,001$. Las mediciones de espectroscopía fotoelectrónica de rayos X (XPS) se realizaron en un equipo Thermo Scientific K-alpha con una fuente de rayos X Al Ka y un analizador hemisférico con doble enfoque de $180^{\circ}$ (XPS, Thermo Fisher Scientific Inc., Waltham, EE. UU.). Las energías de enlace de los fotoelectrones se determinaron asumiendo que la energía de los electrones del carbono 1 s era de $284,7 \mathrm{eV}$.

\section{Evaluación catalítica}

Los materiales sintetizados se evaluaron catalíticamente en la reacción de degradación del glifosato por oxidación húmeda con aire. Se utilizó un reactor de flujo descendente de lecho fijo construido con vidrio de $8 \mathrm{~mm}$ de diámetro y $35 \mathrm{~cm}$ de longitud, operando a temperatura ambiente y presión atmosférica empleando $0,2 \mathrm{~g}$ del catalizador. Se alimentó una solución de glifosato en agua $(15 \mathrm{ppm})$ mediante una bomba de jeringa $(10 \mathrm{ml} / \mathrm{h})$ y se utilizó aire como fuente de oxígeno (30 $\mathrm{ml} / \mathrm{min}$ ). Las muestras fueron recolectadas cada $15 \mathrm{~min}$ (TOS=tiempo de corrida) y se analizaron mediante cromatografía iónica (Dionex ICS-1100, 5890 Serie II Plus, Ion Pac AS18 Anionic Column, AG18 Column Guard y KOH como eluyente). Los productos de degradación se identificaron mediante comparación con patrones cromatográficos.

\section{Resultados}

Los patrones de XRD de ángulo bajo de los catalizadores sintetizados se presentan en la Figura 1A.
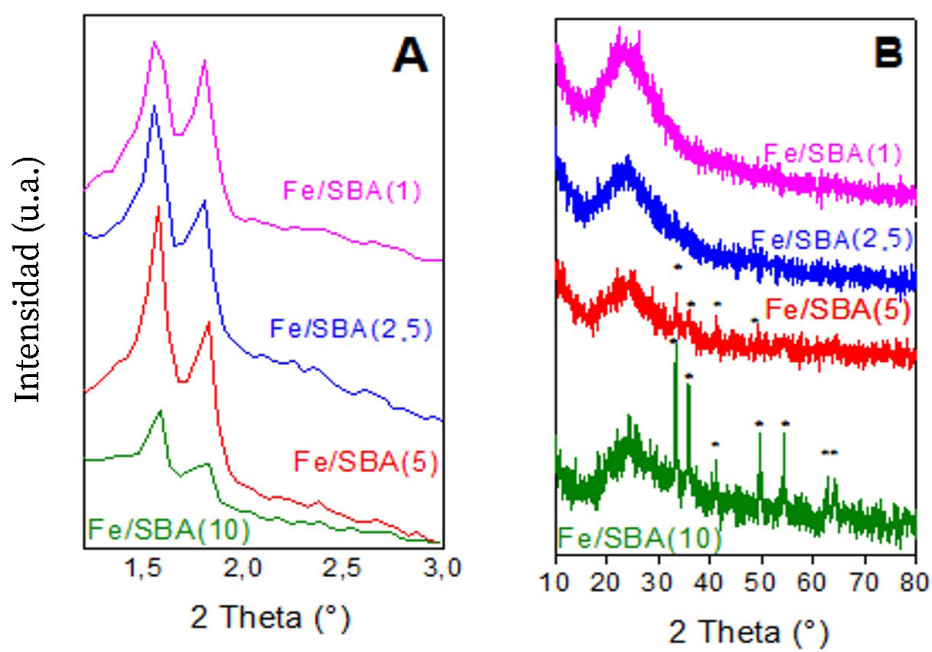

$\left(^{\circ}\right)$ Picos de difracción correspondientes a la fase hematita de a-Fe2O3 Figura 1: XRD A) a bajo ángulo; B) a alto ángulo. 
En todos los casos se observa un patrón de difracción característico de los materiales mesoporosos. Esta disposición se evidencia por la presencia de picos de difracción correspondientes a los planos (110) y (200) que indican una disposición hexagonal de largo alcance en la estructura mesoporosa SBA-15 (Do et al., 2005). Estos resultados concuerdan con los elevados valores áreas específicos obtenidos, del orden de $600-1000 \mathrm{~m}^{2} / \mathrm{g}$ (Tabla 1). Los patrones de difracción de alto ángulo de los materiales sintetizados se observan en la Figura 1B, donde se detecta un pico amplio en un valor de $2 \theta$ entre $20-30^{\circ}$ asignado a la sílice amorfa (Bing Sun et al., 2008).

\begin{tabular}{|c|c|c|c|c|c|c|c|c|c|c|}
\hline \multirow{3}{*}{ Muestras } & \multirow{3}{*}{$\begin{array}{l}\text { Área } \\
\left(\mathrm{m}^{2} / \mathrm{g}\right)\end{array}$} & \multicolumn{9}{|c|}{ Distribución de especies de Fe } \\
\hline & & \multirow{2}{*}{$\begin{array}{c}\mathrm{Fe} \\
(\% \mathrm{p} / \mathrm{p})\end{array}$} & \multicolumn{2}{|c|}{$\begin{array}{l}\mathrm{Fe}^{+3} \text { aislado } \\
(230-300 \mathrm{~nm})\end{array}$} & \multicolumn{2}{|c|}{$\begin{array}{l}\text { Nanoclusters } \\
\text { más pequeños } \\
(300-400 \mathrm{~nm})\end{array}$} & \multicolumn{2}{|c|}{$\begin{array}{l}\text { Nanoclusters } \\
\text { más grandes } \\
(400-500 \mathrm{~nm})\end{array}$} & \multicolumn{2}{|c|}{$\begin{array}{l}\text { Nanopartículas } \\
(>500 \mathrm{~nm})\end{array}$} \\
\hline & & & $\begin{array}{c}\% \\
\text { área } \\
\end{array}$ & $\begin{array}{c}\mathrm{Fe} \\
(\% \mathrm{p} / \mathrm{p})\end{array}$ & $\begin{array}{c}\% \\
\text { área }\end{array}$ & $\begin{array}{c}\mathrm{Fe} \\
(\% \mathrm{p} / \mathrm{p})\end{array}$ & $\begin{array}{c}\% \\
\text { área }\end{array}$ & $\begin{array}{c}\mathrm{Fe} \\
(\% \mathrm{p} / \mathrm{p})\end{array}$ & $\begin{array}{c}\% \\
\text { área }\end{array}$ & $\begin{array}{c}\mathrm{Fe} \\
(\% \mathrm{p} / \mathrm{p})\end{array}$ \\
\hline $\mathrm{Fe} / \mathrm{SBA}(1)$ & 1042 & 0.8 & 39.80 & 0.318 & 42.49 & 0.340 & 17.71 & 0.142 & - & - \\
\hline $\begin{array}{c}\mathrm{Fe} / \\
\text { SBA(2.5) }\end{array}$ & 882 & 2.0 & 25.34 & 0.507 & 36.03 & 0.721 & 38.63 & 0.772 & - & - \\
\hline Fe/SBA(5) & 815 & 4.9 & 22.99 & 1.127 & 30.33 & 1.486 & 42.80 & 2.097 & 3.88 & 0.190 \\
\hline $\mathrm{Fe} / \mathrm{SBA}(10)$ & 632 & 8.3 & 18.62 & 1.545 & 24.95 & 2.071 & 49.85 & 4.138 & 6.58 & 0.546 \\
\hline
\end{tabular}

1 Por ecuación BET b Por método colorimétrico.

Tabla 1: Propiedades fisicoquímicas y distribución relativa de especies de Fe en los sólidos sintetizados.

Las muestras con mayores cargas de Fe presentaron varios picos más intensos y definidos para la muestra de $\mathrm{Fe} / \mathrm{SBA}(10)$. Estos picos corresponden a la presencia de óxidos de hierro formados en el material. Estas líneas de difracción distintivas a 20: 33, 35, 41, 49, 54, 62, 64 ${ }^{\circ}$ se correlacionan con el patrón de difracción a-Fe $\mathrm{O}_{3}$ (Elías et al., 2020a), (Joint Comitte on Powder Diffraction Standars, 1972), (Balu et al., 2010). Los tamaños medios estimados de cristal de $\mathrm{a}-\mathrm{Fe}_{2} \mathrm{O}_{3}$ fueron $21 \pm 4$ y $13 \pm 2 \mathrm{~nm}$ para las muestras $\mathrm{Fe} / \mathrm{SBA}(10)$ y Fe/SBA(5), respectivamente. Teniendo en cuenta que el tamaño medio de los poros del SBA-15 es de $\sim 7 \mathrm{~nm}$ (Elías et al., 2020a), estas nanopartículas deben ubicarse en la superficie externa del soporte (Feng et al., 2004), (Hosseini et al., 2015). Sin embargo, para todas las muestras, no se puede descartar la presencia de especies de óxido de hierro amorfas o con tamaños inferiores al límite de detección.

Por otro lado, la Tabla 1 muestra los resultados del contenido de Fe en cada una de las muestras sintetizadas. Se observa que el porcentaje en peso de heteroátomos cargados en las muestras es proporcional y bastante cercano al contenido nominal de Fe usado en la síntesis correspondiente.

Se aplicó espectroscopía ultravioleta visible con reflectancia difusa (UVvis-DR) para estudiar la naturaleza de las especies de hierro desarrolladas en silicatos SBA-15 modificados con diferentes cargas de Fe utilizando cloruro férrico como precursor metálico. Los espectros ajustados (Figura 2) muestran cuatro regiones de absorción principales (Elías et al., 2011), (Cuello et al., 2015). La región en longitudes de onda más cortas, entre 230 y $300 \mathrm{~nm}$, generalmente se asigna a especies metálicas aisladas (iones $\mathrm{Fe}^{+3}$ ) unidas a átomos de oxígeno presentes en las paredes de silicato. La segunda región, entre 300 y $400 \mathrm{~nm}$, resulta de la absorción de iones Fe presentes en nanoclusters de pequeño tamaño. La tercera región, entre 400 y $500 \mathrm{~nm}$, corresponde a las transiciones de $\mathrm{O}$ a Fe ${ }^{+3}$, cuando los iones metálicos 
están presentes en especies más polimerizadas, formando nanoclusters de óxidos $(\mathrm{FeO})_{n}$ de mayor tamaño. La última región de absorción a longitudes de onda superiores a $500 \mathrm{~nm}$ se atribuye a la transición del par de electrones d-d de iones Fe presentes en nanopartículas de óxido más grandes (probablemente segregadas fuera de los mesocanales y ya evidenciadas por DRX). La Tabla 1 informa los datos obtenidos de los espectros UVvis-DR ajustados y muestra la distribución de especies de Fe en función de la carga de metal. Es importante notar que la capacidad de absorción de los sólidos es consistente con el contenido de metal. Así, los materiales con mayores cargas metálicas presentan señales más intensas en todo el rango de longitudes de onda, así como también un mayor porcentaje en peso de todas las especies. Una proporción mayor (\% de área) de especies de Fe dispersas (iones de Fe aislados y nanoclusters muy pequeños, que absorben a longitudes de onda menores a $400 \mathrm{~nm}$ ) se evidencia para los sólidos modificados con el menor contenido de metales. Ambas contribuciones disminuyen a medida que aumentan las cargas de Fe, siendo evidente la contribución cada vez mayor de nanoagrupamientos más grandes que resultan de la polimerización posterior de las especies aisladas, que habrían comenzado a saturar la superficie.

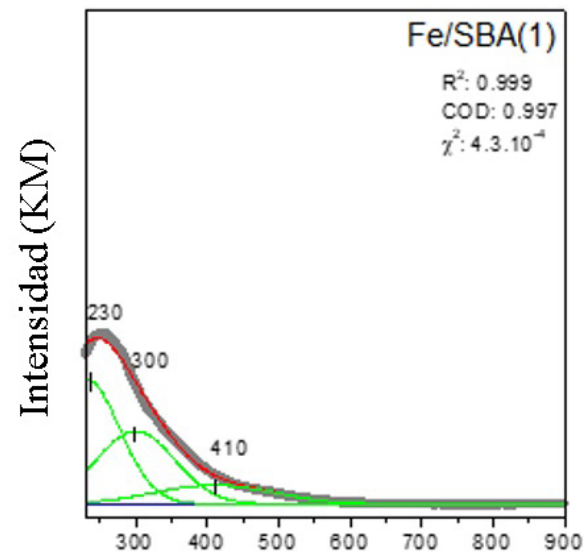

Longitud de onda (nm)

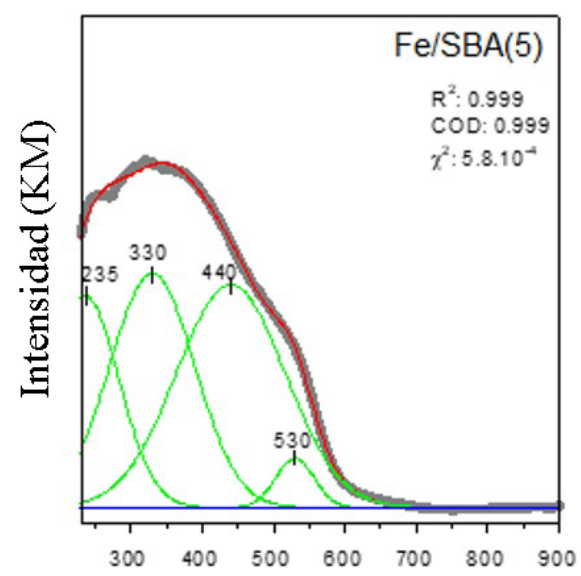

Longitud de onda (nm)

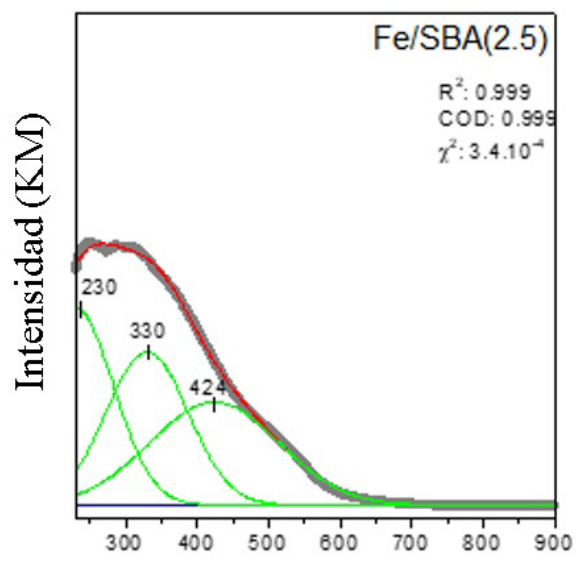

Longitud de onda (nm)

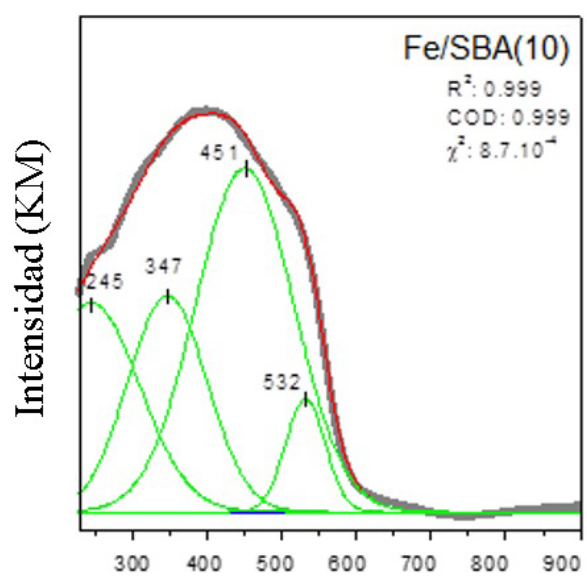

Longitud de onda (nm)

Figura 2: Espectros de UVvis-DR de las muestras sintetizadas. 
Luego, para las muestras con las cargas más altas, la contribución de las especies de hierro segregadas más grandes que absorben a longitudes de onda $>400 \mathrm{~nm}$, nanoclusters de óxido de hierro más grandes y nanopartículas, aumenta notablemente alcanzando porcentajes superiores al $2 \%$ en peso y $0,19 \%$ en peso respectivamente. En concordancia, los patrones de DRX de las muestras con estas cargas más altas mostraron picos correspondientes a la presencia de óxidos de hierro (hematita). Dichas especies, formadas en la superficie externa del soporte, podrían estar bloqueando algunos mesoporos (y en consecuencia aquellas especies más finamente dispersas en su interior) a juzgar por la disminución de los valores de área específica observada para estas muestras.

Los espectros XPS en la zona de Fe 2p para las muestras de Fe/SBA(2.5) y Fe/SBA(10) se muestran en la Figura 3. Es importante notar que la intensidad de las señales aumenta a medida que aumenta la carga de hierro. Asimismo, los picos más anchos observados en comparación con las líneas estrechas características de los clusters de óxidos están dando cuenta de la presencia de especies metálicas más finamente dispersas en la estructura.
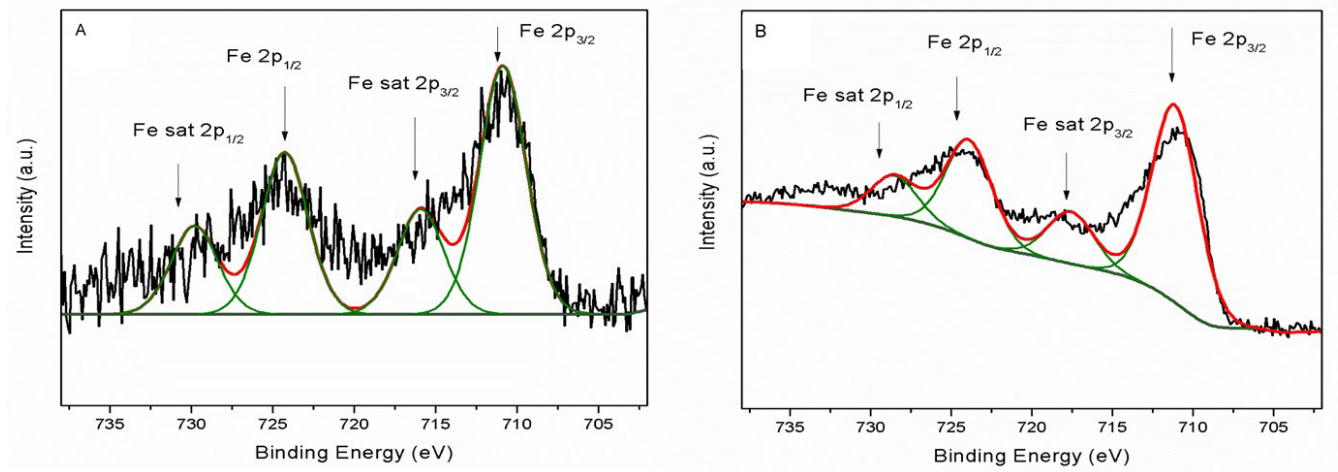

Figura 3: Perfil de fotoelectrones a nivel de núcleo de Fe $2 p$ de las muestras: A) Fe/SBA(2.5) y B) Fe/SBA(10).

Los espectros se podrían ajustar con cuatro picos indicativos del estado químico de $\mathrm{Fe}^{+3}$ en un entorno de óxido. Para ambas muestras, se pudo observar la presencia de un pico de Fe $2 \mathrm{p}_{3 / 2}$ alrededor de $711 \mathrm{eV}$, acompañado de una línea satélite alrededor de 716-717 eV, y de un pico de Fe $2 \mathrm{p}_{1 / 2}$ alrededor de $724 \mathrm{eV}$ con su línea satélite alrededor de $729 \mathrm{eV}$ (Tabla 2). Sin embargo, es interesante notar que la diferencia de energías de enlace entre el pico de $\mathrm{Fe} 2 \mathrm{p}_{3 / 2}$ y su orbital es menor que la encontrada normalmente para una muestra estándar de $\mathrm{a}-\mathrm{Fe}_{2} \mathrm{O}_{3}$ $(\sim 8 \mathrm{eV})$, siendo esta diferencia más acentuada para la muestra de Fe/SBA(2.5) (5 eV).

\begin{tabular}{|c|c|}
\hline Fe/SBA(2.5) & BE(eV) \\
\hline Fe 2p3/2 & 710,88 \\
\hline Fe 2p1/2 & 724,27 \\
\hline Fe sat 2p3/2 & 715,92 \\
\hline Fe sat 2p1/2 & 729,81 \\
\hline Fe/SBA(10) & BE(eV) \\
\hline Fe 2p3/2 & 711,12 \\
\hline Fe 2p1/2 & 723,9 \\
\hline Fe sat 2p3/2 & 717,57 \\
\hline Fe sat 2p1/2 & 728,52 \\
\hline
\end{tabular}

Tabla 2: Valores de energía de unión (BE) de XPS para muestras de Fe/SBA(2.5) y Fe/SBA(10). 
Según Elías et al., 2020a, este desplazamiento de la línea satélite, que indica que las especies de hierro no corresponden a los clusters de hematita, podría dar cuenta de la alta contribución tanto de nanoclusters muy pequeños como de cationes $\mathrm{Fe}^{+3}$ aislados ligados a la superficie de este sólido como fue inferido por UVvis-DR.

Los materiales sintetizados impregnados con hierro se evaluaron catalíticamente en la reacción de degradación del glifosato (Figura 4). Una carga nominal de Fe por encima de $2 \%$ en peso condujo a degradaciones superiores al 70\%. Todos los catalizadores permitieron lograr, en condiciones de presión atmosférica y temperatura ambiente, la fragmentación del herbicida hacia los siguientes iones: acetato, nitrato, nitrito y fosfato (caracterizado por cromatografía líquida iónica).

Se sabe que el glifosato, como muchos otros ácidos fosfónicos, forma complejos estables (1:1 y 1:2) con cationes metálicos divalentes y trivalentes en entornos de coordinación octaédricos y tetraédricos (Caetano et al., 2012), (Subramaniam et al., 1988). En estos complejos, el oxígeno de los grupos fosfonato y carboxilato puede coordinarse con el metal central. Además, el átomo de nitrógeno del grupo amino de la molécula de glifosato también puede participar en coordinación con el metal si no está protonado. Sin embargo, se sabe que el glifosato puede unirse a una superficie sólida, como el suelo, a través de una adsorción específica predominantemente como complejación monodentada y menos comúnmente como complejación bidentada (Li, 2018), (Waiman et al., 2013). Sin embargo, aunque el glifosato puede formar complejos estables con iones Fe (Barja et al., 2001), en nuestras investigaciones, su degradación catalítica podría alcanzarse y evidenciarse por la aparición de sus iones de fragmentación.

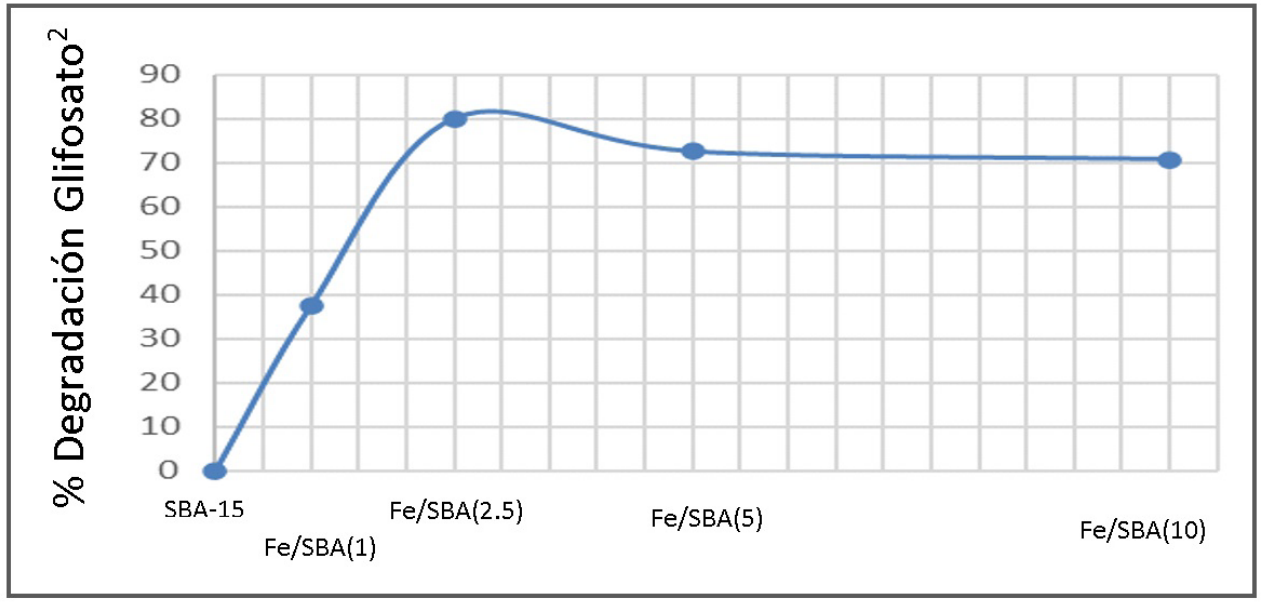

${ }^{2}$ Por cromatografía líquida iónica (TOS = $\left.15 \mathrm{~min}\right)$.

Figura 4: Valores de degradación del glifosato.

Estudios anteriores han informado de que la oxidación catalítica húmeda se produce principalmente a través de reacciones de radicales libres (Kyoung- Hun et al., 2011). El catalizador puede activar moléculas reactivas facilitando la descomposición del contaminante (Sheldon et al., 1981a), (Sheldon et al., 1981b) o participar en un mecanismo de transferencia de oxígeno en el que su función es transferir un átomo de oxígeno a moléculas reactivas 
acuosas mediante complejación y activación (Cavani et al., 1997), (Guo et al., 2003). Como se sabe (Sheldon et al., 1981a), el oxígeno molecular puede ser activado por complejos de metales de transición para participar directamente en la oxidación de compuestos orgánicos. Así, se han propuesto especies intermedias de oxo-hierro (V), formadas a partir de Fe (III), $\mathrm{H}^{+} \mathrm{y}$ $\mathrm{O}_{2}$ como oxidante activo para diferentes reacciones de oxidación conducidas por complejos de hierro (Sheldon et al., 1981a), (Sheldon et al., 1981b).

Por lo tanto, de acuerdo con Sheldon et al., se propone la formación de un complejo de Fe-glifosato en los sólidos de SBA-15 impregnados con hierro. Este complejo activaría el oxígeno molecular y, con el aporte de los protones del medio, se podría generar un intermediario oxo-hierro (V) (Vaschetto et al., 2019). A partir de esto, se produciría la fragmentación del glifosato y la posterior desorción de los productos de degradación, liberando nuevamente los sitios activos del catalizador (Figura 5).

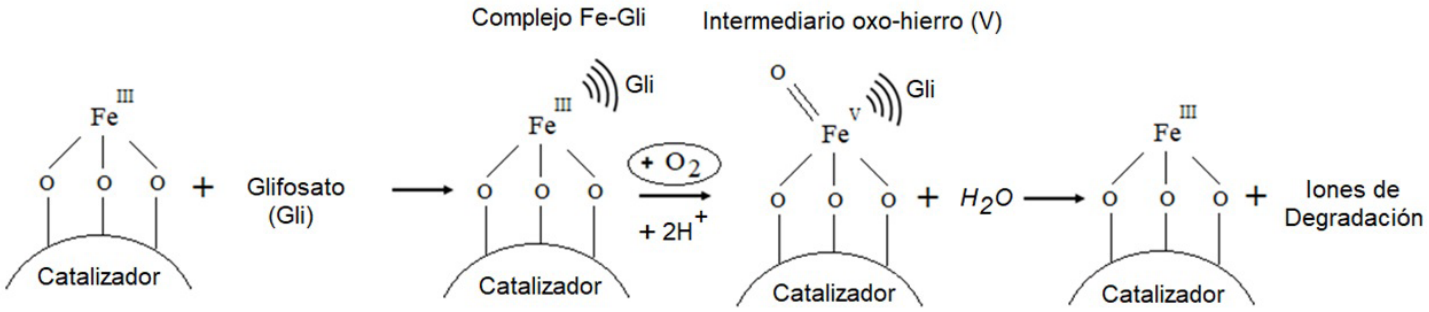

Figura 5: Vía propuesta para la adsorción de glifosato sobre el sólido, formación del complejo, generación del intermediario activo y posterior degradación y desorción de los productos.

Así, los catalizadores modificados con hierro podrían formar un complejo Fe-glifosato (adsorción del sustrato orgánico en la superficie del catalizador) capaz de activar $\mathrm{O}_{2}$, proveniente del flujo de aire, generando un intermediario oxo-hierro (V) que promoverían la degradación del herbicida, en condiciones de reacción suaves. En nuestras condiciones experimentales, la degradación-fragmentación del sustrato se logró con todos los catalizadores modificados con Fe. Sin embargo, el resultado catalítico más favorable (degradación de glifosato de aproximadamente 80\%) se alcanzó utilizando el sólido modificado con un 2,5\% en peso de Fe de carga nominal (Figura 4). Este hecho podría atribuirse a que la carga de metal en la muestra de Fe/SBA(2.5) resulta óptima para alcanzar en la superficie del silicato una alta proporción de especies de Fe aisladas activas, accesibles a las moléculas de reactivo debido a que no serían bloqueadas por las especies de hierro voluminosas (incluso en una proporción muy baja en esta muestra).

Por lo tanto, incluso empleando cloruro de hierro como precursor (cuyo contraión promueve la polimerización de especies, según lo reportado por Elías et al. 2020b), es posible ajustando las variables de síntesis para lograr una gran cantidad de especies de Fe finamente dispersas, altamente eficiente para la degradación de contaminantes.

Para mayores contenidos de Fe (5 y 10\% en peso) las especies metálicas más segregadas de mayor tamaño (nanoclusters de óxido y nanopartículas) desarrolladas en la estructura, podrían bloquear los sitios activos evitando así la degradación del herbicida. Para un contenido de Fe más bajo (1\% en peso), la cantidad de especies de Fe aisladas no sería suficiente para lograr una mayor degradación del sustrato. Finalmente, también se evaluó el material puro SBA-15; no se observó degradación de glifosato lo que indica que el soporte solo no es activo, y la presencia de Fe es clave para llevar a cabo la degradación.

Un aspecto importante a estudiar en un proceso catalítico es la estabilidad del catalizador 
y la posibilidad de reciclado. Así, se llevaron a cabo tres ciclos experimentales con $\mathrm{Fe} /$ $\mathrm{SBA}(2,5)$. Después de cada reacción, se recuperó el catalizador, se calcinó a $500{ }^{\circ} \mathrm{C}$, se pesó y luego se reutilizó. La degradación del glifosato mostró el mismo comportamiento en los tres ciclos catalíticos, demostrando la estabilidad del sólido y la posibilidad de reutilización. Cabe señalar que se determinó una diferencia insignificante en la masa del catalizador después de cada ciclo, lo que sugiere que la presencia de especies no volátiles adsorbidas en la superficie del catalizador que podrían envenenar los sitios activos, es insignificante.

\section{Conclusión}

Los nanomateriales del tipo SBA-15 se modificaron con éxito con hierro. Se obtuvieron estructuras muy ordenadas, propias de materiales mesoporosos, con áreas específicas del orden de 600-1000 m2/g. Estos catalizadores se evaluaron en la reacción de degradación-fragmentación del glifosato mediante oxidación catalítica húmeda con aire. La mayor degradación del glifosato (80\%) se logró utilizando el material Fe/SBA(2,5), el cual presentó una alta proporción de especies de Fe accesibles aisladas que, al actuar como agentes complejantes, favorecerían la formación de complejos con glifosato (Fe-Gli). Dicho complejo sería capaz de activar el oxígeno molecular dando lugar al intermediario activo oxo-hierro (V), altamente oxidante para producir la fragmentación del sustrato en iones que incluyen acetato, nitrato, nitrito y fosfato. De esta forma, la degradación del herbicida en iones de cadena corta, menos tóxicos y más biodegradables, puede tener lugar en condiciones de presión atmosférica y temperatura ambiente. Así, se propone un proceso de remediación de aguas residuales contaminadas con glifosato que implica un menor impacto ambiental y una mayor sostenibilidad. 


\section{Referencias}

Dubois, A. y Lacouture, L. (2011). "Bilan de présence des micropolluants dans les milieux aquatiques continentaux. Période 2007-2009”. Commissariat général au développement durable, 54, (2011). https://side.developpement-durable.gouv.fr/Default/doc/ SYRACUSE/213231

Barja, B., Herszage, J. y dos Santos Afonso, M. (2001). “Iron (111)-phosphonate complexes”. Polyhedron, 20, (15-16), 1821-1830.

Robert, D. y Malato, S. (2002). "Solar photocatalysis: a clean process for water detoxification”. Science of the Total Environment, 291, (1-3), 85-97.

Kyoung-Hun, K. y Son-Ki, I. (2011). "Heterogeneous catalytic wet air oxidation of refractory organic pollutants in industrial wastewaters: A review". Journal of Hazardous Materials, 186, (1), 16-34.

Elías, V., Ochoa Rodriguez, P., Vaschetto, E., Pecchi, G., Huck-Iriart, C., Casuscelli, S. y Eimer, G. (2020). "Tailoring the stability and photo-Fenton activity of Fe-modified nanostructured silicates by tuning the metal speciation from different synthesis conditions". Molecular Catalysis, 481, 110217.

Elías, V., Sabre, E., Sapag, K., Casuscelli, S. y Eimer, G. (2012). "Influence of the Cr loading in $\mathrm{Cr} / \mathrm{MCM}-41$ and $\mathrm{TiO}_{2} / \mathrm{Cr} / \mathrm{MCM}-41$ molecular sieves for the photodegradation Acid Orange 7”. Applied Catalysis A: General, 413, 280-291.

Patterson, A. (1939). “The Scherrer Formula for X-Ray particle size determination”. Physical Review Journals Archive, 56, (10), 978-982.

Greenberg, A., Clesceri, L. y Eaton, A. American public health association, American Water Works Association, Water Pollution Control Federation (1992). Standard methods for the examination of water and wastewater. Washington, DC: Joint Editorial Board.

Neyens, E. y Baeyens, J. (2003). "A review of classic Fenton's peroxidation as an advanced oxidation technique”. Journal of Hazardous Materials, 98, (1-3), 33-50.

Do, Y., Kim, J., Park, J., Park, S., Hong, S., Suh, C. y Lee, G. (2005). "Photocatalytic decomposition of 4-nitrophenol on Ti-containing MCM-41”. Catalysis Today, 101, 299-305.

Bing Sun, L., Hui Kou, J., Chun, Y., Yang, J., Na Gu, F., Wang, Y., Hua Zhu, J., Gang Zou, Z. (2008). New Attempt at Directly Generating Superbasicity on Mesoporous Silica SBA-15”. Inorganic Chemistry, 47,4199-4208.

Joint Committee on Powder Diffraction Standars (1972). "Joint Committee on Powder Diffraction Standards 330664”. Analytical Chemistry, 44, 12, 75A.

Balu, A., Pineda, A., Yoshida, K., Campelo, J., Gai, P., Luque, R. y Romero, A. (2010). "Sinergia $\mathrm{Fe} / \mathrm{Al}$ en nanopartículas de $\mathrm{Fe}_{2} \mathrm{O}_{3}$ soportadas sobre materiales de aluminosilicato porosos: Excelentes actividades en reacciones de oxidación”. Chemical Communication, 46, $7825-7827$.

Feng, J., Hu, X. y Yue, P. (2004). "Discoloration and Mineralization of Orange II Using Different Heterogeneous Catalysts Containing Fe: A Comparative Study”. Environmental Science \& Technology, 38, 5773-5778. 
Hosseini, S., Ahmadi, R., Ghavi, A. y Kashi, A. (2015) "Synthesis and characterization of a- $\mathrm{Fe}_{2} \mathrm{O}_{3}$ mesoporous using SBA-15 silica as template and investigation of its catalytic activity for thermal decomposition of ammonium perchlorate particles". Powder Technology, 278, 316-322.

Elías, V., Vaschetto, E., Sapag, K., Oliva, M., Casuscelli, S. y Eimer, G. (2011). "MCM-41 based materials for the photo-catalytic degradation on Acid Orange 7”. Catalysis Today,172, (1), 58-65.

Cuello, N., Elías, V., Crivello, M., Torres, C., Oliva, M. y Eimer, G. (2015). "Development of iron modified MCM-41 as promising nano-composites with specific magnetic behavior". Microporous and Mesoporous Materials, 203, 106-115.

Caetano, M., Ramalho, T., Botrel, D., Da Cunha, E. y Carvalho de Mello, W. (2012). "Understanding the inactivation process of organophosphorus herbicides: a DFT study of glyphosate metallic complexes with $\mathrm{Zn}^{+2}, \mathrm{Ca}^{+2}, \mathrm{Mg}^{+2}, \mathrm{Cu}^{+2}, \mathrm{Co}^{+3}, \mathrm{Fe}^{+3}, \mathrm{Cr}^{+3}$ and $\mathrm{Al}^{+3}$ ". International Journal of Quantum Chemistry, 112, (15), 2752-2762.

Coutinho, C. y Mazo, L. (2005). “Complexos Metálicos com o Herbicida Glifosato: Revisão”. Química Nova, 28, (6), 1038-1045.

Harris, W., Sammons, R., Grabiak, R., Mehrsheikh, A. y Bleeke, M. (2012). "Computer Simulation of the Interactions of Glyphosate with Metal Ions in Phloem”. Journal of Agricultural and Food Chemistry, 60, (24), 6077-6087.

Subramaniam, V. y Hoggard, P. (1988). "Metal complexes of glyphosate”. Journal of Agricultural and Food Chemistry, 36, (6), 1326-1329.

Li, H. (2018) "Degradation of glyphosate by Mn-oxides: mechanisms, pathways, and source tracking”, Doctoral Dissertation. University of Delaware, United States.

Sheals, J., Sjöberg, S. y Persson, P. (2002). “Adsorption of Glyphosate on Goethite: Molecular Characterization of Surface Complexes”. Environmental Science \& Technology, 36, (14), 3090-3095.

Waiman, C. V., Avena, M. J., Regazzoni, A. E. y Zanini, G. P. (2013). "A real time in situ ATRFTIR spectroscopic study of glyphosate desorption from goethite as induced by phosphate adsorption: effect of surface coverage”. Journal of Colloid and Interface Science, 394, 485-489.

Sheldon, R. A. y Kochi, J. K. (1981a). Metal-Catalyzed Oxidations of Organic Compounds: Mechanistic Principles and Synthetic Methodology Including Biochemical Processes. London: Academic Press.

Sheldon, R. A. y Kochi, J. K. (1981b). Metal-Catalyzed Oxidations of Organic Compounds in the Liquid Phase: A Mechanistic Approach. London: Academic Press.

Cavani, F. y Trifiro, F. (1997). "Classification of industrial catalysts and catalysis for the petrochemical industry”. Catalysis Today, 34, (3-4), 269-279.

Guo, J. y Al-Dahhan, M. (2003). "Kinetics of wet air oxidation of phenol over a novel catalyst”. Industrial \& Engineering Chemistry Research, 42, (22), 5473-5481.

Vaschetto, E., Sicardi, M., Elías, V., Ferrero, G., Carraro, P., Casuscelli, S. y Eimer, G. (2019). "Metal modified silica for catalytic wet air oxidation (CWAO) of glyphosate under atmospheric conditions". Adsorption, 1-8. 
Elías, V., Benzaquén, T., Ochoa Rodríguez, P., Cuello, N., Tolley, A. y Eimer, G. (2020). "Elucidating Iron Speciation Tuned by Handling Metal Precursor for more Efficient Designing of Nanostructured Fenton Catalysts”. Catalysis Letters, 150, 196-208. 